\title{
Potential drug-drug interactions in inpatients treated at the Internal Medicine ward of Tikur Anbessa Specialized Hospital
}

This article was published in the following Dove Press journal:

Drug, Healthcare and Patient Safety

14 August 2017

Number of times this article has been viewed

\section{Zelalem Tilahun Tesfaye' Teshome $\mathrm{Nedi}^{2}$ \\ 'Department of Clinical Pharmacy, School of Pharmacy, College of Medicine and Health Sciences, University of Gondar, Gondar, ${ }^{2}$ Department of Pharmacology and Clinical Pharmacy, College of Health Sciences, Addis Ababa University, Addis Ababa, Ethiopia}

Video abstract

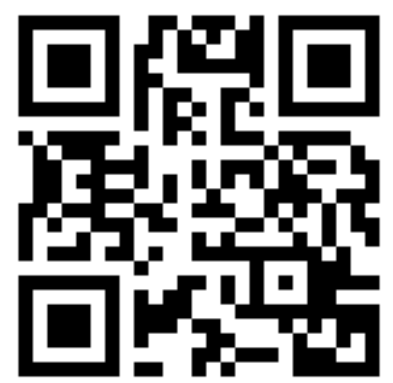

Point your SmartPhone at the code above. If you have a QR code reader the video abstract will appear. Or use: http://youtu.be//SX3FFGLDVM
Correspondence: Zelalem Tilahun Tesfaye

Department of Clinical Pharmacy, School of Pharmacy, College of Medical and Health Sciences, University of Gondar, PO Box 196, Gondar, Ethiopia Tel +25I 9I 3824722

Email zelalemII@yahoo.com
Purpose: Although the concomitant use of multiple drugs often increases therapeutic effectiveness, certain combinations result in unwanted drug-drug interactions (DDIs). Most interactions go unnoticed by physicians due to the absence of new clinical signs and symptoms, and because they often produce a worsening of already existing symptoms. Quantification of the occurrence of the potential DDIs is essential to prevent the harmful effects associated with interactions. This study was launched to assess the prevalence of potential DDIs in the Internal Medicine ward of Tikur Anbessa Specialized Hospital, Addis Ababa, Ethiopia.

Patients and methods: Cross-sectional data were gathered from the medical charts of 252 randomly selected patients who were admitted to the Internal Medicine ward during August 23 to October 23, 2013, and exposed to at least two concomitant drugs. Potential DDIs were identified using Medscape Drug Interaction Checker. The data were analyzed using SPSS software. Logistic regression analysis was used to determine the presence of association between variables and $p$-value $<0.05$ was considered statistically significant.

Results: At least one potential DDI was found in $78.2 \%$ of the patients. The mean number of potential interactions per patient was $3.7 \pm 3.4$. Out of the 719 potential interactions identified, $49.8 \%$ were pharmacokinetic type, $44.6 \%$ were pharmacodynamic and the remaining $5.6 \%$ were unknown mechanisms. Major potential DDIs accounted for $13.1 \%$ of the whole interactions; $53.5 \%$ were moderate interactions; and the remaining $33.4 \%$ were minor interactions. Ceftriaxone, cimetidine and heparin were the three most involved drugs in major potential interactions. Prescription of five or more concomitant drugs was associated with high risk of encountering potential DDIs.

Conclusion: The findings of this study showed that the prevalence of potential DDIs among inpatients was high. Pharmacists should closely review drugs prescribed for patients and avoid dispensing combinations of drugs that may have serious DDIs.

Keywords: concomitant drugs, potential drug interactions, Tikur Anbessa Specialized Hospital, Ethiopia, pharmacokinetic interactions, pharmacodynamic interactions

\section{Introduction}

A drug-drug interaction (DDI) is an event that occurs when the effects of a drug are modified by another drug that is taken concomitantly. ${ }^{1}$ DDIs can be classified, according to the mechanisms by which drugs interact with each other, as pharmaceutical, pharmacokinetic and pharmacodynamic. ${ }^{2}$ DDIs may result in either increase or decrease in efficacy, in treatment failure, or in an increased toxicity of medications. ${ }^{3,4}$ However, not all potential DDIs are clinically significant. ${ }^{5}$ 
Although the concurrent use of multiple drugs often increases therapeutic effectiveness, certain combinations are harmful. ${ }^{6}$ Most interactions go unnoticed by physicians due to the absence of new clinical signs and symptoms and because they often produce a worsening of already existing symptoms. ${ }^{7}$ DDIs sometimes go undetected because of a lack of knowledge and training, low motivation and poor attitude. ${ }^{8}$ The incidence of potential DDIs may be affected by different factors, such as higher number of concomitant drugs, presence of comorbidities and advanced age. ${ }^{9-11}$

Although the presence of multiple physicians involved in patient management increases the quality of care through teamwork, it may also result in multiple separate prescriptions if there is low communication between the prescribers, which in turn increases the risk of inappropriate drug combinations. ${ }^{7}$ The presence of multiple comorbidities may increase the number of medications required for treatment of the conditions and associated DDIs. ${ }^{10}$ Because Tikur Anbessa Specialized Hospital (TASH) is a teaching hospital, patients are examined by many interns and residents, and as the largest referral hospital in Ethiopia, patients with a higher degree of morbidity and associated multiple comorbidities, from all over the country, are referred to this hospital, which may increase the risk of potential DDIs for patients treated at TASH.

Studies on the subject of DDIs have never been conducted in TASH before. Considering these facts and the fact that there were no clinical pharmacists in inpatient settings of the hospital during the study period, to detect and manage potential DDIs, it is possible to predict that DDIs may be prevalent in the inpatient settings, especially in the Internal Medicine ward since the largest number of patients with a wide range of disease conditions are admitted to the ward. This study was launched to determine the prevalence of potential DDIs in the Internal Medicine ward of TASH.

\section{Patients and methods}

The study was conducted in TASH, a tertiary hospital located in Addis Ababa, the capital of Ethiopia. The study was conducted in Internal Medicine ward from August 23 to October 23, 2013.

Cross-sectional data were gathered from the medical charts of 252 randomly selected patients who were admitted to the ward during the study period and exposed to at least two concomitant drugs. If patients who were included earlier in the study came back to the ward later during the study period, they were excluded.

The data collected included age and sex of the patients, their primary diagnoses, presence or absence of comorbidities and the list of medications prescribed concurrently. Poten- tial DDIs were identified using Medscape Drug Interaction Checker, which is an online reference database accessed from reference.medscape.com/drug-interactionchecker. The database contains drug information updated through systematic review of major medical and pharmacy journals, US Food and Drug Administration (FDA) announcements and practice guidelines. In addition to information from the database, patient factors were also considered while recording interactions. Thus, potential DDIs identified by the database would not be recorded if measures were taken by prescribers (eg, modification of dose, route of administration and so on) upon prescription to prevent those potential interactions. Identified interactions were classified by severity as major, moderate and minor, as well as based on underlying mechanisms as pharmacokinetic, pharmacodynamic and unknown mechanisms. The data were analyzed using SPSS software v16 (SPSS Inc., Chicago, IL, USA). Logistic regression analysis was used to determine the presence of association between variables and $p$-value $<0.05$ was considered as statistically significant.

Letter of ethical clearance was obtained from the Ethics Committee of the Department of Internal Medicine ofTASH. The need for patient consent was waived by the approving committee provided privacy and confidentiality were ensured during review of patients' charts by data collectors. Thus, the name and address of patients were not recorded in the data collection forms.

\section{Results}

\section{Sociodemographic and clinical data}

Of the total 252 patients, 136 (54.0\%) were males and 116 (46.0\%) were females. The patients' age ranged from 13 years to 87 years, and the mean age was $39.5 \pm 18.0$ years. The most frequent diagnoses were infectious diseases, cardiovascular diseases and hematologic disorders (Table 1).

\section{Prevalence of potential DDIs}

A total of 1280 medications were prescribed for 252 patients. The minimum number of concomitant drugs per patient was two and the maximum was 13 . The mean number of drugs prescribed per subject was $5.1 \pm 2.2$. In $20.6 \%$ of patients, four concomitant drugs were prescribed. The most-prescribed drugs were ceftriaxone (123 patients), followed by furosemide (80 patients), cimetidine (59 patients) and heparin (54 patients). Figure 1 shows the 15 most-prescribed drugs for the study subjects.

Among the 252 patients, 197 had prescriptions with at least one potential DDI, producing a prevalence value of $78.2 \%$. Of these patients with potential DDIs, $52.8 \%$ were male and the remaining were female. 
Table I Sociodemographic and clinical data of patients

\begin{tabular}{|c|c|}
\hline Variables & Frequency (\%) \\
\hline \multicolumn{2}{|l|}{ Sex, n } \\
\hline Male & $136(54)$ \\
\hline Female & $116(46)$ \\
\hline Age, years, mean \pm SD & $39.5 \pm 18.0$ \\
\hline$<15$ & $10(4.0)$ \\
\hline $15-24$ & 47 (18.7) \\
\hline $25-44$ & $98(38.9)$ \\
\hline $45-64$ & $66(26.2)$ \\
\hline$>65$ & $31(12.3)$ \\
\hline \multicolumn{2}{|l|}{ Disease type } \\
\hline Infectious & $58(23.0)$ \\
\hline Cardiovascular & $52(20.6)$ \\
\hline Hematologic & $43(17.1)$ \\
\hline Solid tumor & $5(2.0)$ \\
\hline Renal disease & $12(4.8)$ \\
\hline Thromboembolic disorders & $9(3.6)$ \\
\hline Gastrointestinal disorders & $6(2.4)$ \\
\hline Neurologic & $7(2.8)$ \\
\hline Liver disease & $9(3.6)$ \\
\hline Diabetes mellitus & $8(3.1)$ \\
\hline Stroke & $22(8.7)$ \\
\hline Airway obstruction & $6(2.4)$ \\
\hline Other* & $15(5.9)$ \\
\hline \multicolumn{2}{|l|}{ Comorbidities } \\
\hline Present & $154(61.1)$ \\
\hline Absent & $98(38.9)$ \\
\hline Number of drugs per patient, mean \pm SD & $5.1 \pm 2.2$ \\
\hline $2-3$ & $65(25.8)$ \\
\hline $4-5$ & $90(35.7)$ \\
\hline $6-7$ & $69(27.4)$ \\
\hline $8-9$ & $15(5.9)$ \\
\hline$>9$ & $13(5.1)$ \\
\hline
\end{tabular}

Note: *Ascites $(n=3)$, intracranial lesion $(n=3)$, brain abscess $(n=2)$, lung abscess $(n=2)$, systemic lupus erythematosus $(n=2)$, thyrotoxicosis $(n=1)$, cystic fibrosis $(n=l)$ and pleural effusion $(n=l)$.
Out of the 1280 drugs prescribed to patients, 719 pairs were identified to have potential interactions. The mean DDI in these patients was 3.7 \pm 3.4 . Almost one-third of the patients with identified potential DDIs $(31.0 \%)$ had only one interaction.

\section{Types of potential DDls}

Half of all DDIs identified in patients were pharmacokinetic interactions (358/719), whereas nearly the entire other half comprised pharmacodynamic interactions (321/719). The remaining few were interactions with unknown mechanisms (40/719).

Out of 358 pharmacokinetic DDIs identified, $67.6 \%$ were associated with one drug affecting the metabolism of the other. Interactions associated with altered excretion showed the next higher prevalence among pharmacokinetic interactions (19.8\%), followed by altered distribution $(9.5 \%)$ and absorption (3.1\%) respectively (Figure 2).

\section{Severity of potential DDIs}

Assessment of severity of the identified DDIs was thereafter performed. Out of the identified DDIs, 94 (13.1\%) were major interactions (Table 2). More than half of the DDIs (53.5\%) were moderate interactions, and the remaining 240 (33.4\%) were minor interactions. The mean number of major DDIs per patient was found to be 1.6 \pm 1.0 . Nearly two-thirds (38/58) of patients with major potential interactions had only one DDI.

A total of 123 active substances were prescribed for the 252 patients incorporated in the study, five of which were not found in the Medscape drug interaction database. Hence,

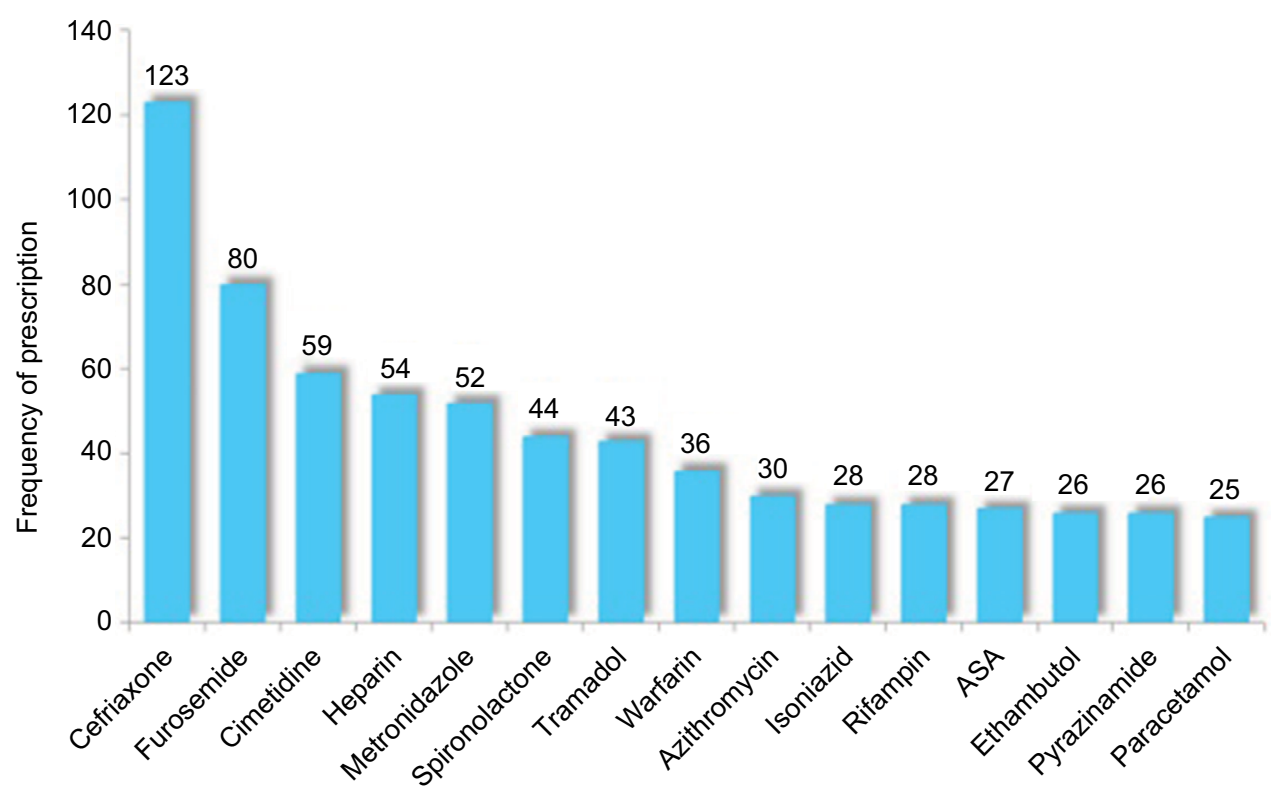

Figure I The most-prescribed drugs among inpatients treated at the Internal Medicine ward of Tikur Anbessa Specialized Hospital. Abbreviation: ASA, acetylsalicylic acid 
analysis was performed for $\sim 96 \%$ of the drugs. Thus, 27 active substances accounted for 94 major DDIs. The drugs most involved in the major DDIs were ceftriaxone $(30.1 \%)$, cimetidine $(30.1 \%)$ and heparin $(30.1 \%)$, followed by

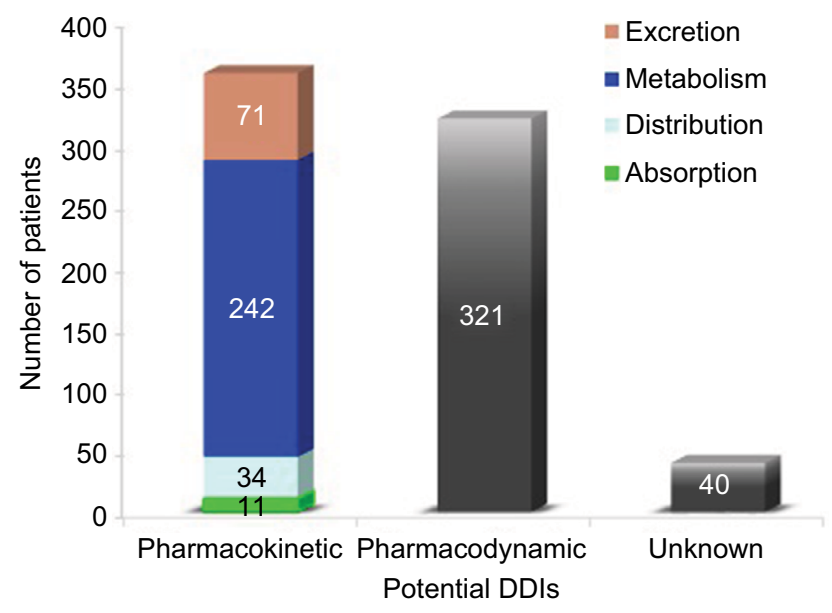

Figure 2 Types of potential drug-drug interactions (DDIs) based on the mechanism of interaction identified in patients. warfarin $(28.7 \%)$. When the drug classes were compared, anticoagulants were found in $54.2 \%$ of major DDIs and antibiotics were found in $52.1 \%$.

\section{Factors associated with potential DDls}

Both univariate and multivariate analyses showed that patients taking more than five concomitant drugs are at higher risk of DDIs $(p=0.000)$. Both sex $(p=0.660)$ and advanced age $(p=0.511)$ were not significantly associated with exposure to DDIs (Table 3).

\section{Discussion}

When hospitalized patients take multiple medications, DDIs are encountered frequently. In some cases, these interactions cause adverse reactions and changes in therapeutic efficacy. This study gives important information regarding the prevalence of potential DDIs in the medical ward of TASH, their types based on the mechanism of interaction and level of severity.

Table 2 Drugs involved in major potential drug interactions

\begin{tabular}{|c|c|c|c|c|}
\hline $\begin{array}{l}\text { Perpetrator } \\
\text { drug }\end{array}$ & Object drug & Frequency (\%) & Mechanism of interaction & Potential outcome \\
\hline Ceftriaxone & Heparin & $22(23.4)$ & Pharmacodynamic synergism & Increased risk of bleeding \\
\hline Ceftriaxone & Warfarin & $7(7.4)$ & Pharmacodynamic synergism & Increased risk of bleeding \\
\hline Heparin & Warfarin & $7(7.4)$ & Pharmacodynamic synergism & Increased risk of bleeding \\
\hline Cimetidine & Dexamethasone & $6(6.4)$ & Inhibition of metabolism by CYP3A4 & Cushing's syndrome, adrenal insufficiency \\
\hline Cimetidine & Prednisolone & $5(5.2)$ & Inhibition of metabolism by CYP3A4 & Cushing's syndrome, adrenal insufficiency \\
\hline Cimetidine & Simvastatin & $5(5.2)$ & Inhibition of metabolism by CYP3A4 & Liver damage, skeletal muscle injury (rare) \\
\hline Cimetidine & Warfarin & $5(5.2)$ & Inhibition of metabolism by CYP3A4 & Increased risk of bleeding \\
\hline Metronidazole & Simvastatin & $4(4.2)$ & Inhibition of metabolism by CYP3A4 & Liver damage, skeletal muscle injury (rare) \\
\hline Cimetidine & Hydrocortisone & $3(3.2)$ & Inhibition of metabolism by CYP3A4 & Cushing's syndrome, adrenal insufficiency \\
\hline Furosemide & Gentamicin & $3(3.2)$ & Pharmacodynamic synergism & Risk of nephrotoxicity, ototoxicity \\
\hline Azithromycin & Digoxin & $3(3.2)$ & Increased absorption & Digoxin toxicity \\
\hline Spironolactone & $\mathrm{KCl}$ & $3(3.2)$ & Pharmacokinetic synergism & Hyperkalemia \\
\hline Cimetidine & Atorvastatin & $3(3.2)$ & Inhibition of metabolism by CYP3A4 & Severe myopathy \\
\hline Sulfamethoxazole & Warfarin & $3(3.2)$ & $\begin{array}{l}\text { Competition for plasma protein binding, } \\
\text { decreased metabolism }\end{array}$ & Increased risk of bleeding \\
\hline Efavirenz & Warfarin & $2(2.1)$ & Inhibition of metabolism by CYP2C9/10 & Increased risk of bleeding \\
\hline Dexamethasone & Simvastatin & $\mathrm{I}(\mathrm{I} . \mathrm{I})$ & Increased metabolism by CYP3A4 & Liver damage, skeletal muscle injury (rare) \\
\hline Omeprazole & Digoxin & $\mathrm{I}(\mathrm{I} . \mathrm{I})$ & Increased absorption & Digoxin toxicity \\
\hline Allopurinol & Warfarin & $\mathrm{I}(\mathrm{I} . \mathrm{I})$ & Decreased metabolism & Increased risk of bleeding \\
\hline Cimetidine & Cyclosporine & $\mathrm{I}(\mathrm{I} . \mathrm{I})$ & Inhibition of metabolism by CYP3A4 & Risk of nephrotoxicity and neurotoxicity \\
\hline Testosterone & Cyclosporine & $\mathrm{I}(\mathrm{I} . \mathrm{I})$ & Decreased metabolism & Risk of nephrotoxicity and neurotoxicity \\
\hline Sulfamethoxazole & Cyclosporine & $\mathrm{I}(\mathrm{I} . \mathrm{I})$ & Unknown mechanism & Increased nephrotoxicity \\
\hline Rifampin & Warfarin & $\mathrm{I}(\mathrm{I} . \mathrm{I})$ & Increased metabolism by CYP3A4 & Decreased effect \\
\hline Amiodarone & Azithromycin & $\mathrm{I}(\mathrm{I} . \mathrm{I})$ & Pharmacodynamic synergism & QT interval prolongation \\
\hline Amiodarone & Warfarin & $\mathrm{I}(\mathrm{I} . \mathrm{I})$ & Inhibition of metabolism by CYP2C9 & Increased risk of bleeding \\
\hline Furosemide & Paromomycin & $\mathrm{I}(\mathrm{I} . \mathrm{I})$ & Pharmacodynamic synergism & Increased toxicity \\
\hline Nifedipine & Simvastatin & $\mathrm{I}(\mathrm{I} . \mathrm{l})$ & Inhibition of metabolism by CYP3A4 & Liver damage \\
\hline Cimetidine & Clopidogrel & $\mathrm{I}(\mathrm{I} . \mathrm{I})$ & Inhibition of metabolism by CYP2CI9 & Decreased effect \\
\hline Efavirenz & Clopidogrel & $\mathrm{I}(\mathrm{I} . \mathrm{I})$ & Inhibition of metabolism by CYP2CI9 & Decreased effect \\
\hline Total & & $94(100)$ & & \\
\hline
\end{tabular}

Abbreviation: CYP, cytochrome P450. 
Table 3 Binary logistic analysis for factors associated with potential drug-drug interactions

\begin{tabular}{|c|c|c|c|c|}
\hline \multirow[t]{2}{*}{ Variables } & \multicolumn{2}{|c|}{ Exposure to DDIs } & \multicolumn{2}{|l|}{ OR $(95 \% \mathrm{Cl})$} \\
\hline & Exposed & Not exposed & Crude & Adjusted $^{\#}$ \\
\hline \multicolumn{5}{|l|}{ Sex } \\
\hline Male & 104 & 32 & $0.804(0.439, \mathrm{I} .47 \mathrm{I})$ & $0.864(0.450,1.659)$ \\
\hline Female & 93 & 23 & 1.00 & 1.00 \\
\hline \multicolumn{5}{|l|}{ Age, years } \\
\hline$<50$ & 139 & 40 & 1.00 & 1.00 \\
\hline$\geq 50$ & 58 & 15 & $1.113(0.571,2.170)$ & $1.272(0.620,2.6 \mathrm{II})$ \\
\hline \multicolumn{5}{|c|}{ Number of medications } \\
\hline$<5$ & 72 & 45 & 1.00 & 1.00 \\
\hline$\geq 5$ & 125 & 10 & $7.812(3.7 \mid 2,16.44 I)$ & $7.890(3.739,16.65 \mathrm{I})^{*}$ \\
\hline
\end{tabular}

Notes: "Adjusted for sex, age and number of medications; *significant. Bold represents significant values $(p<0.05)$.

Abbreviations: $\mathrm{OR}$, odds ratio; $\mathrm{Cl}$, confidence interval; DDI, drug-drug interactions.

The prevalence of potential DDIs in this study was $78.2 \%$. This figure agrees with the findings of a study in Romania, which reported a prevalence value of $78.03 \% .{ }^{12}$ Much lower prevalence values were reported by studies conducted in different settings in Thailand (27.9\%) and Iran (20.3\%). ${ }^{13,14}$ This broad difference in prevalence of potential DDIs may be explained by factors, such as difference in availability of alternative drugs and absence of clinical pharmacists and drug information software to provide drug information in the inpatient settings of TASH during the study period. Involvement of pharmacists in reviewing hospitalized patients' medication prescriptions would significantly reduce the prevalence and harmful effects of DDIs. Pharmacists contribute to better treatment outcome by early detection of medication errors, by monitoring treatment outcomes and by recommending treatment modification ${ }^{15-17}$

In the present study, pharmacokinetic interactions show a little predominance over pharmacodynamic interactions, which is probably because of the high prescription frequency of drugs that affect liver enzymes, such as cimetidine and metronidazole.

In this study, the prevalence of major DDIs was $13.1 \%$. This finding agrees with findings of studies conducted previously in other countries such as Italy and Denmark, as well as a study conducted in a different hospital in Ethiopia. ${ }^{18-20}$ Significantly higher incidence of major interactions was recorded in two studies conducted in other tertiary hospitals of the country ${ }^{21,22}$ This wide difference may be due to the difference in study population in the studies. The first study was conducted on cardiac patients, whereas the second study was on psychiatric patients, both of which involved chronic diseases treated with multiple medications.

In the present study, two-thirds of the identified DDIs come under either major or moderate category. Major DDIs are those that may be life threatening or may cause permanent damage and hence not recommended for concomitant use, whereas moderate interactions are those that may cause deterioration of patient condition, warranting close monitoring. In contrast, minor interactions have limited clinical effect and usually require no intervention. Patients with identified major and moderate DDIs should be monitored for the potential outcomes of the interactions. These patients may need modification or alteration of therapy or dose adjustment. One-third of the potential DDIs in this study were minor interactions. These are DDIs that produce changes on effects of drugs not sufficient to precipitate clinically significant outcomes. However, minor DDIs can sometimes produce significant adverse outcomes on special populations, such as the elderly or patients with various organ failures. Close monitoring is needed for such patients even for minor DDIs. The class of drugs that were prescribed and had the largest number of major DDIs was the anticoagulants, followed by antibiotics. In this study, the frequency of involvement of drugs in major DDIs corresponds with their frequency of prescription.

No statistically significant differences were found between men and women regarding exposure to potential DDIs. Age is another important variable to consider in relation to DDIs. In this study, no statistically significant association was found between age and exposure to DDIs. The absence of association between sex and exposure to DDIs may be because the finding only indicates the potential interactions, which may be highly dependent on prescription factors than on patient factors. This explanation could also be applied for the absence of association between age and exposure to DDIs. It should be noted that although the risk of exposure to potential DDIs is similar across different age groups, the risk of actual occurrence of the interactions and the degree of morbidity due to them might not be similar.

Patients on five or more concomitant drugs were found to have higher rate of exposure to potential DDIs than those 
taking less than five drugs. This is because a higher number of concomitant medications increases the probability of each drug encountering another drug with potential interaction.

The limitations of the study should not be overlooked. First, the interactions found were only potential; the presence of potential interactions does not always mean that the interactions actually occurred in the patients. The study does not attempt to determine whether the interactions actually occurred. Second, this study only covered the Internal Medicine ward, which makes the results difficult to apply for other wards such as the Pediatric ward. Despite these limitations, the findings of this study can be useful as input for understanding the extent of the problem and taking measures to improve the practice of managing drug interactions.

\section{Conclusion}

The findings of this study showed that the prevalence of potential DDIs among inpatients was high. Most of the interactions were of minor-to-moderate severity. Major DDIs are considered clinically important and should be avoided by health care professionals. Pharmacists, in particular, should closely review drugs prescribed for patients and avoid dispensing combinations of drugs that may have serious DDIs. DDI software should be introduced in the workstations of pharmacists.

\section{Disclosure}

The authors report no conflicts of interest in this work.

\section{References}

1. Nidhi S. Concept of drug interaction. Int Res J Pharm. 2012;3:120-122.

2. Bolhuis MS, Panday PN, Pranger AD, et al. Pharmacokinetic drug interactions of antimicrobial drugs: a systematic review on oxazolidinones, rifamycines, macrolides, fluoroquinolones, and beta-lactams. Pharmaceutics. 2011;3(4):865-913.

3. Bjornsson T, Callaghan J, Einolf H, et al; Pharmaceutical Research and Manufacturers of America (PhRMA) Drug Metabolism/Clinical Pharmacology Technical Working Group; FDA Center for Drug Evaluation and Research (CDER). The conduct of in vitro and in vivo drug-drug interaction studies: a pharmaceutical research and manufacturers of America (PhRMA) perspective. Drug Met Dispos. 2003;31(7):815-832.

4. Mozayani A, Raymon L, editors. Handbook of Drug Interactions: A Clinical and Forensic Guide. 1st ed. Totowa, NJ: Humana Press; 2004.
5. Goldberg RM, Mabee J, Chan L, et al. Drug-drug and drug disease interactions in ED: analysis of high risk population. Am J Emerg Med. 1996;14(5):450-477.

6. Teixeira J, Crozatti M, Santos C, Lieber N. Potential drug-drug interactions in prescriptions to patients over 45 years of age in primary care, Southern Brazil. PLoS One. 2012;7(10):e47062.

7. Picazo J, Ruiz J, Sanchez J, et al. Prevalence and typology of potential drug interactions occurring in primary care patients. Eur J Gen Pract. 2010;16(2):92-99.

8. Jindal D, Sharma H, Karim S, et al. Drug interactions in prescriptions from selected Indian community pharmacies. J Pharm Pract Res. 2005;35:332.

9. Sivva D, Mateti UV, Neerati VM, Thiruthopu NS, Martha S. Assessment of drug-drug interactions in hypertensive patients at a superspeciality hospital. Perspect Clin Res. 2015;6(1):58-61.

10. Kapp PA, Klop AC, Klop AC, Jenkins LS. Drug interactions in primary health care in the George subdistrict, South Africa: a cross-sectional study. S Afr Fam Pract. 2013;55(1):78-84.

11. Mateti UV, Lalwani T, Nagappa AN, et al. Assessment of drug-related problems in depressive patients. Perspect Clin Res. 2015;6(1):56-61.

12. Bucşa C, Farcaş A, Cazacu I, et al. How many potential drug-drug interactions cause adverse drug reactions in hospitalized patients? Eur J Int Med. 2013;24(1):27-33.

13. Janchawee B, Wongpoowarak W, Owatranporn T, Chongsuvivatwong V. Pharmacoepidemiologic study of potential drug interactions in outpatients in a university hospital in Thailand. J Clin Pharm Ther. 2005;30(6):13-20.

14. Seperhi G, Khazaelli P, Dahooje F, Seperhi E, Dehghani M. Prevalence of potential drug interactions in an Iranian general hospital. Indian $J$ Pharm Sci. 2012;74(1):75-79.

15. Lopez-Martin C, Garrido SM, Alcaide-Garcia J, Faus FV. Role of clinical pharmacists to prevent drug interactions in cancer outpatients: a single center experience. Int J Clin Pharm. 2014;36(6):1251-1259.

16. Leape LL, Cullen DJ, Clapp MD, et al. Pharmacist participation on physician rounds and adverse drug events in the intensive care unit. JAMA. 1999;281(3):267-270.

17. Kaboli PJ, Hoth AB, McClimon BJ, Schnipper JL. Clinical pharmacists and inpatient medical care. Arch Intern Med. 2006;166(9):955-964.

18. Magro L, Conforti A, Del Zotti F, et al. Identification of severe potential drug-drug interactions using an Italian general-practitioner database. Eur J Clin Pharmacol. 2008;64(3):303-309.

19. Juurlink D, Mamdani M, Kopp A, Laupacis A, Redelmeier DA. Drugdrug interactions among elderly patients hospitalized for drug Toxicity. JAMA. 2003;289(13):1652-1658.

20. Bhagavathula AS, Birhane A, Tigistu H, et al. Prevalence of potential drug-drug interactions among internal medicine ward in University of Gondar Teaching Hospital, Ethiopia. Asian Pac JTrop Biomed. 2014;4(1): 204-208.

21. Chelkeba L, Alemseged F, Bedada W. Assessment of potential drug-drug interactions among outpatients receiving cardiovascular medications at Jimma University Specialized Hospital, South West Ethiopia. Int $J$ Basic Clin Pharmacol. 2013;2:144-152.

22. Mezgebe HB, Seid K. Prevalence of potential drug-drug interactions among psychiatric patients in Ayder referral hospital. J Sci Innov Res. 2015;4(2):71-75.
Drug, Healthcare and Patient Safety

\section{Publish your work in this journal}

Drug, Healthcare and Patient Safety is an international, peer-reviewed open access journal exploring patient safety issues in the healthcare continuum from diagnostic and screening interventions through to treatment, drug therapy and surgery. The journal is characterized by the rapid reporting of reviews, original research, clinical, epidemiological and

\section{Dovepress}

post-marketing surveillance studies, risk management, health literacy and educational programs across all areas of healthcare delivery. The manuscript management system is completely online and includes a very quick and fair peer-review system. Visit http://www.dovepress.com/ testimonials.php to read real quotes from published authors. 